\title{
Polygonatum sibiricum polysaccharides prevent LPS-induced acute lung injury by inhibiting inflammation via the TLR4/Myd88/NF-кB pathway
}

\author{
TIAN-YIN LIU, LI-LI ZHAO, SHI-BIAO CHEN, BEN-CHAO HOU, \\ JIAN HUANG, XIU HONG, LIAN QING, YU FANG and ZHE TAO \\ Department of Anesthesia, The First Affiliated Hospital of Nanchang University, Nanchang, Jiangxi 330006, P.R. China
}

Received September 5, 2019; Accepted February 5, 2020

DOI: $10.3892 /$ etm.2020.9097

\begin{abstract}
Inflammation plays an important role in cases of acute lung injury (ALI), and the Toll-like receptor 4/nuclear factor- $\kappa \mathrm{B}(\mathrm{TLR} 4 / \mathrm{NF}-\kappa \mathrm{B})$ pathway, which can be regulated by Polygonatum sibiricum polysaccharides (PSPs), is closely related to the dynamics of lipopolysaccharide (LPS)-induced inflammation. Thus, we sought to evaluate whether or not PSPs prevent LPS-induced ALI by way of inhibiting inflammation via the TLR4/NF- $\mathrm{B}$ pathway in rats. We established an ALI rat model by tracheal instillation of LPS, and by pre-injection of PSPs into rats to examine PSPs in the ALI rat model. We found that PSPs attenuated LPS-induced lung pathological changes in ALI rats, decreased LPS-induced myeloperoxidase (MOP) activity, and elevated malondialdehyde (MDA) levels in lung tissue. However, PSPs also decreased the LPS-induced increase in the neutrophil ratio, and decreased inflammatory factor levels in bronchoalveolar lavage fluid (BALF). Moreover, PSPs decreased LPS-induced increases in inflammatory factors measured by mRNA expression, and altered the levels of expression of TLR4, medullary differentiation protein 88 (Myd88), p-IKB- $\alpha / \mathrm{IKB}-\alpha$ and $\mathrm{p}-\mathrm{p} 65 / \mathrm{p} 65$ proteins in lung tissue. In vitro, PSPs also reduced apoptosis induced by LPS in BEAS-2B cells by suppressing
\end{abstract}

Correspondence to: Dr Li-Li Zhao, Department of Anesthesia, The First Affiliated Hospital of Nanchang University, 17 Yongwaizheng Street, Nanchang, Jiangxi 330006, P.R. China

E-mail: zhaolltop@163.com

Abbreviations: ALI, acute lung injury; LPS, lipopolysaccharide; SIRS, systemic inflammatory response; PSPs, Polygonatum sibiricum polysaccharides; MOP, myeloperoxidase; MDA, malondialdehyde; BALF, bronchoalveolar lavage fluid; RT-qPCR, Real-time fluorescence quantification PCR; LBP, lipopolysaccharide binding

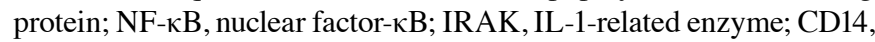
cluster of differentiation 14; TLR4, Toll-like receptor 4; MyD88, medullary differentiation protein 88

Key words: Polygonatum sibiricum polysaccharides, lipopolysaccharide, acute lung injury, TLR4, NF- $\mathrm{B}$ inflammation through its effect of inhibiting the TLR4/NF- $\mathrm{B}$ pathway. In conclusion, our results suggest that PSPs may be a potential drug for effective treatment of LPS-induced ALI, due to the ability to inhibit inflammation through effects exerted on the TLR4/Myd88/NF- $\kappa$ B pathway.

\section{Introduction}

Acute lung injury (ALI) is a type of a pulmonary inflammatory response syndrome and is mainly characterized by increased alveolar capillary permeability and alveolar epithelial damage. In the process of ALI, acute, persistent alveolar-capillary membrane damage results in large amounts of protein extravasations and induces the formation of a transparent membrane in the alveolar space (1). In addition, with an imbalance in ventilated blood flow, lung compliance typically declines, and resultant hypoxemia and dyspnea often occur in patients with ALI. ALI is a commonly encountered critical type of emergency in clinical practice, with a high mortality rate ranging from 50 to $70 \%(2,3)$. While the pathogenesis of ALI is not fully elucidated, previous research indicates that uncontrolled systemic inflammatory responses (SIRS) caused by varied factors can induce the onset and progression of ALI, especially as the lung is the foremost organ most susceptible to such types of inflammatory damage $(4,5)$. Furthermore, it is certain that the imbalance between pro-inflammatory responses and anti-inflammatory responses is one of the main causes of the onset and progression of ALI $(6,7)$.

Polygonatum sibiricum polysaccharides (PSPs) mainly composed of four chemically distinct monosaccharides, were originally extracted from the dried rhizome of Polygonatum, a genus of flowering plants. Recent modern pharmacological research suggests that PSPs have many biological activities including antioxidant, anti-aging, anti-fatigue, immune enhancement, antibacterial, and anti-inflammatory activities $(8,9)$. In addition, previous research has indicated that PSPs can exert antitumor effects by inhibiting the transmission of the Toll-like receptor 4/mitogen-activated protein kinase/ nuclear factor- $\kappa \mathrm{B}$ (TLR4-MAPK/NF- $\kappa \mathrm{B}$ ) signaling pathway (10). The TLR4 signaling pathway has also been found to be closely related to the dynamics underlying ALI $(11,12)$. TLR4 is expressed in immune cells such as macrophages and neutro- 
phils, and in non-immune cells such as alveolar endothelial cells and epithelial cells. Infiltration of alveolar inflammatory cells is the key to the inflammatory response, and endothelial cell damage is the basis of structural degradation of the alveolar basement membrane in ALI $(13,14)$. Moreover, previous research has suggested that TLR4-mediated inflammation plays a key role in lipopolysaccharide (LPS)-induced ALI (15), and plays a key role in kidney injury (16).

TLR is a transmembrane transduction receptor for LPS signaling from extracellular to intracellular space. Furthermore, TLR4 can directly bind to LPS, and binds to lipopolysaccharide-binding protein (LBP)-LPS-cluster of differentiation 14 (CD14), thereby activating the NF- $\mathrm{BB}$ signaling pathway through its impact upon dynamics underlying medullary differentiation protein 88 (Myd88). This leads to the synthesis and release of various inflammatory mediators, and finally initiates and amplifies the inflammatory response $(17,18)$. However, whether or not PSPs are able to prevent LPS-induced ALI by inhibiting inflammation via the TLR4/NF- $\kappa B$ pathway is unclear. Therefore, in the present study, we sought to examine and answer the above question.

\section{Materials and methods}

Animals and treatment. We used Wistar rats (5-6 weeks of age; weight, 130-180 g) to establish animal-based models for the present research. In this study, total of 50 rats (male: female=1:1) were all purchased from Shanghai SLAC Laboratory Animal Co., Ltd. Rats were fed daily, housed at $22 \pm 2^{\circ} \mathrm{C}$, and we used a $12 \mathrm{~h}$ light/dark cycle at our experimental center. Rats were randomly assigned to five treatment groups, including the: Control group, LPS group, L-PSP group, M-PSP group, and H-PSP group. The LPS group was composed of the ALI rats model without PSP treatment. The ALI rat model was composed of rats that were first intraperitoneally injected with normal saline. After $30 \mathrm{~min}$, we used $1 \mathrm{mg} / \mathrm{kg}$ lipopolysaccharide (LPS, purity $>95 \%$, cat. no. L2880, Sigma-Aldrich; Merck KGaA) which was intraperitoneally injected. After $16 \mathrm{~h}$, lipopolysaccharide ( $3 \mathrm{mg} / \mathrm{kg}$ ) was instilled into the trachea, and $1 \mathrm{~h}$ later, the rats were intraperitoneally injected with normal saline. In total, $2 \mathrm{~h}$ prior to the establishment of the ALI model, rats in the L-PSP group, the M-PSP group, and the H-PSP group were injected intraperitoneally with PSPs (20 mg/kg for L-PSP, $40 \mathrm{mg} / \mathrm{kg}$ for M-PSP, $80 \mathrm{mg} / \mathrm{kg}$ for H-PSP). PSP was purchased from Ci Yuan Biotechnology Co., Ltd., and the purity was $>98 \%$. Saline was used to dissolve the PSPs. In addition, all rats in this study were anesthetized by intraperitoneal injection of $50 \mathrm{mg} / \mathrm{kg}$ pentobarbital sodium before sacrifice.

All animal protocols were reviewed and approved by The Animal Care and Use Committee of The First Affiliated Hospital of Nanchang University (Nanchang, Jiangxi, China) and conformed to standard guidelines from The National Institution of Health (19).

Hematoxylin and eosin $(H \& E)$ staining. Frozen slices of rat lung were slightly dried, stained with hematoxylin for $3 \mathrm{~min}$ at room temperature, stained with eosin for $10 \mathrm{sec}$ at room temperature, dehydrated using an ascending ethanol gradient, made transparent and then sealed with neutral gum.
Histopathological changes were observed under light microscopy (magnification, x100; CKX41; Olympus Corporation) and imaged for assessment.

Lung wet/dry (W/D) weight, myeloperoxidase (MOP) and malondialdehyde levels. Rats were anesthetized by intraperitoneal injection of $50 \mathrm{mg} / \mathrm{kg}$ pentobarbital sodium before euthanasia, before the rats were euthanized by $\mathrm{CO}_{2}$ as previously described (20). Rats were sacrificed to obtain lung tissues, which were drained and measured for wet weight. Afterwards, lung tissues were incubated at $80^{\circ} \mathrm{C}$ for $4 \mathrm{~h}$ and then tissues were weighed to obtain measures of dry weight. Measures of activity of MOP and the levels of malondialdehyde (MDA) were measured as previously described $(21,22)$.

Inflammatory cytokines and cell counting assay. Rats were sacrificed and bronchoalveolar lavage fluid (BALF) was collected. Neutrophils and total cell numbers in BALF samples were counted as previously described $(21,22)$. Rat tumor necrosis factor (TNF) $\alpha$ (cat. no. ab100785, Abcam), interleukin (IL)-1 $\beta$ (cat. no. ab100768, Abcam), IL-6 (cat. no. ab100772, Abcam) and IL-8 (cat. no. SBJ-R0033, Nanjing Biological) ELISA kits were used to detect the levels of inflammatory cytokines in BALF samples.

Reverse transcription-quantitative PCR. TNF- $\alpha$, IL-1 $\beta$, L-6, and IL-8 levels in samples from rats were measured as previously described (23) by using fluorescence based real-time quantitative PCR (RT-qPCR) and gene-specific TaqMan primer/probe sets on a ABI Prism 7000 Sequence Detection System (Applied Biosystems). GAPDH mRNA transcription was used for internal loading control. PCR primer sets were composed as follows: TNF- $\alpha-F$, 5'-CTGAACTTCGGGGTGATCGG-3' and TNF- $\alpha-\mathrm{R}$, 5'-GGCTTGTCACTCGAATTTTGAGA-3'; IL-1 $\beta$-F, 5'-GAAATGCCACCTTTTGACAGTG-3' and IL-1 $\beta-\mathrm{R}$, 5'-TGGATGCTCTCATCAGGACAG-3'; IL-6-F, 5'-TCTATA CCACTTCACAAGTCGGA-3' and IL-6-R, 5'-GAATTG CCATTGCACAACTCTTT-3'; IL-8-F, 5'-TCGAGACCA TTTACTGCAACAG-3' and IL-8-R, 5'-CATTGCCGGTGG AAATTCCTT-3'.

Western blot analysis. Protein levels were analyzed by using western blotting as previously described (23) and GAPDH protein transcription factor was used for an internal loading control. All antibodies were purchased from Abcam with the corresponding information as follows: anti-TLR4 (cat. no. ab22048, dilution 1:2,000), anti-MyD88 (cat. no. ab107585, dilution 1:3,000), anti-IкB- $\alpha$ (cat. no. ab32518, dilution 1:500), anti-IкB- $\alpha$ (phospho) (cat. no. ab92700, dilution 1:500) anti-NF-kB p65 (acetyl K310) (cat. no. ab19870, dilution 1:300), or anti-p65 phospho S636 (cat. no. ab86299, dilution 1:5,000), or anti-GAPDH (cat. no. ab9484, dilution 1:3,000).

Flow cytometry detection of apoptosis. Normal unafflicted human lung epithelial cells (BEAS-2B) (CRL-9609; ATCC) were cultured using DMEM (cat. no. 12491-15, Thermo Fisher Scientific, Inc.) at a temperature of $37^{\circ} \mathrm{C}$ and in a constant atmosphere of $5 \% \mathrm{CO}_{2}$. In the LPS treatment group, only $24 \mathrm{~h}$ of LPS stimulation was permitted, but for the PSP (1.5 g/l) 

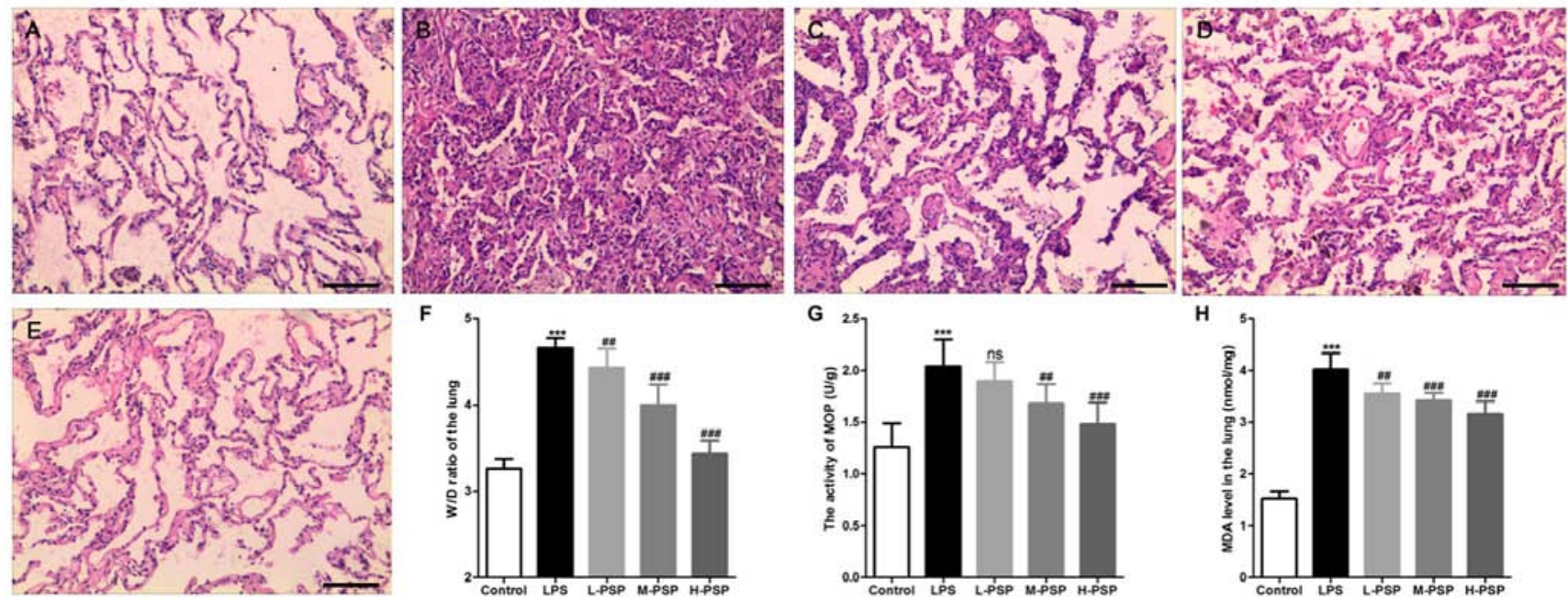

Figure 1. Effect of PSPs on LPS-induced pathological changes in the lungs in ALI rats. (A-E) Hematoxylin and eosin (H\&E) staining was used to observe pathological changes in the lungs of (A) control group, (B) LPS group, (C) L-PSP group, (D) M-PSP group and (E) H-PSP group of rats. (F) The value of the W/D in lung tissues of the different groups. (G) MOP activity in lung tissues of the different groups. (H) The MDA level in lung tissues of the different groups. A total of 10 rats in each group, each indicator for each rat was tested at least 3 times independently; ${ }^{* * *} \mathrm{P}<0.001$ vs. the control group; NS, not significant at $\mathrm{P}>0.05 ;{ }^{\# \#} \mathrm{P}<0.01$ and ${ }^{\# \# \#} \mathrm{P}<0.001$ vs. the LPS group. Scale bar, $100 \mu \mathrm{m}$. PSPs, Polygonatum sibiricum polysaccharides; ALI, acute lung injury; LPS, lipopolysaccharide; MOP, myeloperoxidase; MDA, malondialdehyde; W/D, wet/dry weight ratio. Groups: L-PSP, 20 mg/kg; M-PSP, 40 mg/kg; H-PSP, 80 mg/kg PSPs.

treatment group, PSP was administered $1 \mathrm{~h}$ before LPS stimulation in the PSP+LPS group. BEAS-2B cells were collected $24 \mathrm{~h}$ post-LPS stimulation, and then the Annexin V FITC/PI kit (Invitrogen; Thermo Fisher Scientific, Inc.) was used for flow cytometry to detect apoptosis. Beckman CytoFLEX Flow cytometry (Beckman Coulter, Inc.) was used to analyze levels of cell apoptosis.

Statistical analysis. We used SPSS v20.0 software (IBM Corp.) to analyze data. The Student's t-tests were used to compare differences between two treatment groups. One-way ANOVA was used to compare differences between multiple groups and the corresponding Tukey post-hoc test was used. $\mathrm{P}<0.05$ was considered to indicate a statistically significant difference at which the null hypothesis of no differences among treatment groups was rejected.

\section{Results}

PSPs attenuate LPS-induced lung injury in ALI rats. We used $\mathrm{H} \& \mathrm{E}$ staining to detect pathological changes in lungs. As exemplified in Fig. 1, the alveolar structure of the control group was intact, the alveolar cavity was clearly visible, and there was no obvious exudation (Fig. 1A). However, we observed alveolar septal thickening, massive inflammatory cell infiltration in the interstitial and alveolar spaces, telangiectasia, hemorrhaging, alveolar insufficiency, or observed alveolar fusion in some areas (Fig. 1B). Additionally, the results indicated that PSPs significantly attenuated LPS-induced histopathological changes and that this effect occurred in a dose-dependent manner (Fig. 1C-E). In addition, data related to pathological changes in the lung tissues demonstrated that PSPs reduced LPS-induced increases in lung tissue wet/dry weight ratio (W/D) (Fig. 1F), reduced LPS-induced increases in MOP activity (Fig. 1G) and elevation in levels of MDA (Fig. 1H).
Similarly, these functions of PSPs were found to have occurred in a dose-dependent manner.

PSPs attenuate LPS-induced pulmonary inflammation in ALI rats. Excessive or uncontrolled inflammation is an underlying cause of ALI, and neutrophils play an important role in associated dynamics. Thus, we used BALF tests to assess measures of lung inflammation in the different treatment groups of rats, and found that PSPs were able to attenuate LPS-induced increases in neutrophil ratios (Fig. 2A) and attenuated levels of inflammatory factors in BALF (Fig. 2B-E). Moreover, PSPs were able to reduce LPS-induced increases in mRNA expression of inflammatory factors in lung tissues of the ALI-afflicted rats (Fig. 2F).

PSPs inhibit activation of the TLR4/NF- $\kappa B$ pathway in lung tissue of ALI rats. The TLR4/NF- $\mathrm{B}$ pathway is known to be closely related to the dynamics of LPS-induced inflammation. Thus, we measured the expression levels of key proteins in the TLR4/NF- $\mathrm{B}$ pathway by using western blotting. As shown in Fig. 3, LPS was able to induce elevated levels of TLR4, Myd88, p-IKB- $\alpha / \mathrm{IKB}-\alpha$, and p-p65/p65 proteins in lung tissue of ALI afflicted rats. In contrast, the expression levels of TLR4, Myd88, p-IKB- $\alpha /$ IKB- $\alpha$, and p-p65/p65 proteins in lung tissue of the H-PSP rat treatment group were significantly decreased when compared with the levels in the LPS treatment group (Fig. 3).

PSPs reduce LPS-induced apoptosis in human normal lung epithelial cells in vitro. We cultured normal unafflicted human lung epithelial cells (BEAS-2B cells) in vitro, added LPS to the culture medium, and detected apoptosis by using flow cytometry. As shown in Fig. 4A, LPS was able to significantly increase the proportion of cells that underwent apoptosis in the BEAS-2B cell treatment group, and PSPs 
A
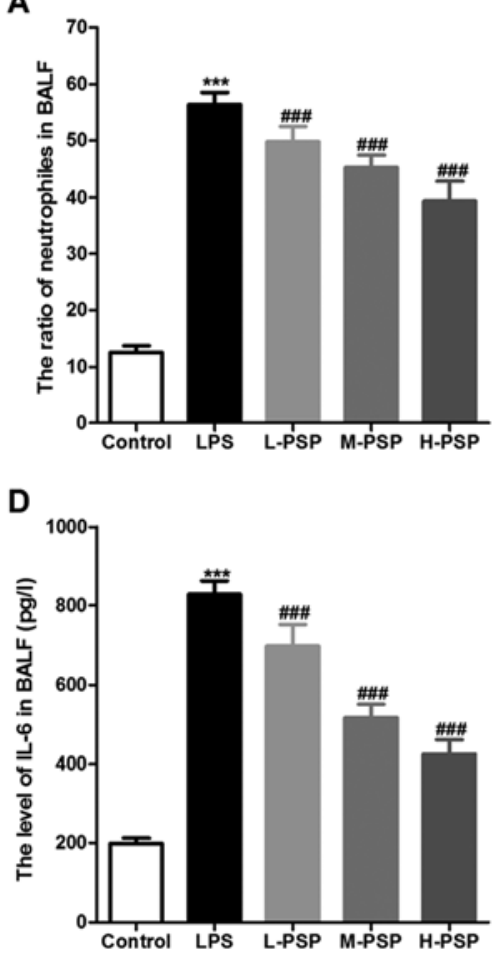

B

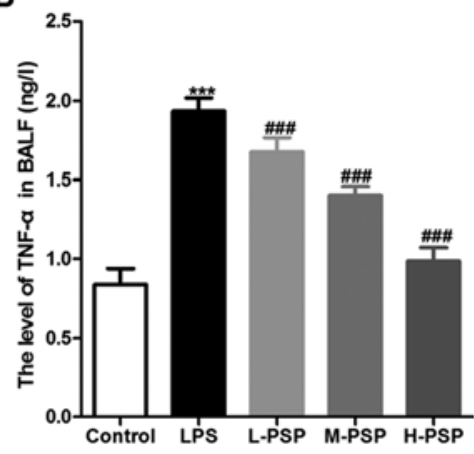

E

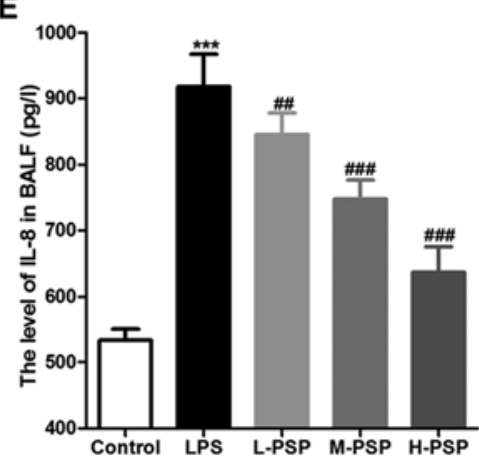

C

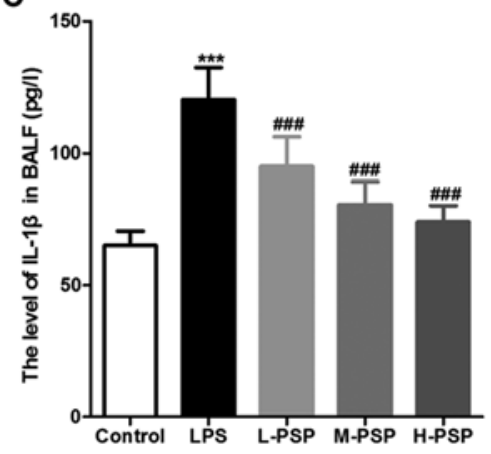

$\mathbf{F}$

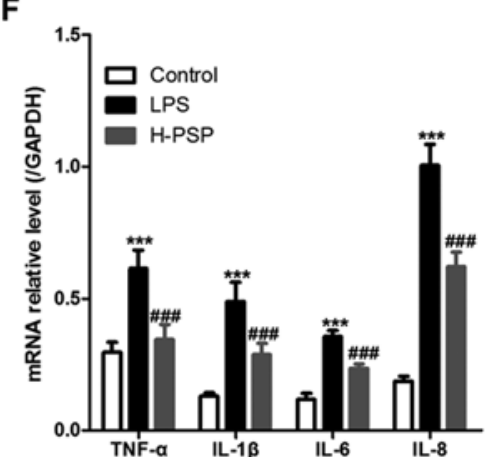

Figure 2. Effect of PSPs on neutrophil ratio and inflammatory factor content in BALF of the ALI rats. (A) Proportion of neutrophils in BALF of the different groups of rats. (B) TNF- $\alpha$, (C) IL-1 $\beta$, (D) IL-6 and (E) IL-8 levels in BALF of the different groups of rats. (F) mRNA expression of TNF- $\alpha$, IL-1 $\beta$, IL-6 and IL-8 in lung tissues of the different groups of rats. A total of 10 rats in each group, each indicator for each rat was tested at least 3 times independently; ${ }^{* * *} \mathrm{P}<0.001$ vs. the control group; ${ }^{\# \#} \mathrm{P}<0.01$ and ${ }^{\# \# \#} \mathrm{P}<0.001$ vs. the LPS group. PSPs, Polygonatum sibiricum polysaccharides; LPS, lipopolysaccharide; BALF, bronchoalveolar lavage fluid; ALI, acute lung injury; TNF- $\alpha$, tumor necrosis factor- $\alpha$; IL, interleukin. Groups: L-PSP, 20 mg/kg; M-PSP, 40 mg/kg; H-PSP, $80 \mathrm{mg} / \mathrm{kg}$ PSPs.
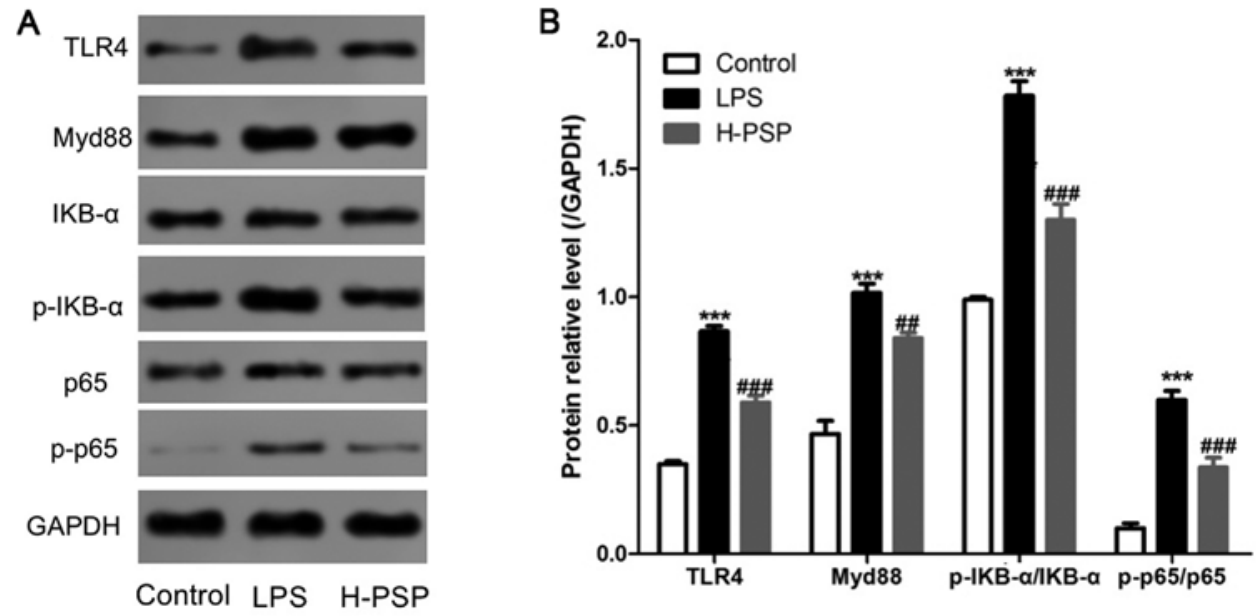

Figure 3. Effect of PSPs on the TLR4/NF-кB pathway in lung tissue of ALI rats. (A) Western blot analysis was used to detect the expression of proteins in lung tissues of the different group of rats: control group, LPS group and H-PSP group. (B) Statistical analysis of the western blotting. A total of 10 rats in each group, each indicator for each rat was tested at least 3 times independently; ${ }^{* * *} \mathrm{P}<0.001$ vs. the control group; ${ }^{\# \#} \mathrm{P}<0.01$ and ${ }^{\# \# \#} \mathrm{P}<0.001$ vs. the LPS group. PSPs,

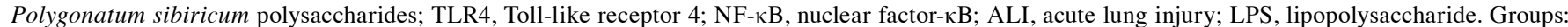
L-PSP, $20 \mathrm{mg} / \mathrm{kg}$; M-PSP, $40 \mathrm{mg} / \mathrm{kg}$; H-PSP, $80 \mathrm{mg} / \mathrm{kg}$ PSPs.

significantly reduced the proportion of apoptosis induced by LPS in the BEAS-2B cells. The mRNA expression levels of inflammatory factors were assessed using RT-qPCR, and the results indicated that PSPs could also reduce LPS-induced increases in mRNA expression levels of inflammatory factors in BEAS-2B cells in vitro (Fig. 4B). Furthermore, we measured the levels of expression of key proteins in the TLR4/NF- $\kappa$ B pathway by using western blotting and found that PSPs were also able to reduce LPS-induced increases in the expression levels of TLR4, Myd88, p-IKB- $\alpha / \mathrm{IKB}-\alpha$, and $\mathrm{p}-\mathrm{p} 65 / \mathrm{p} 65$ proteins in BEAS-2B cells cultured in vitro (Fig. 4C and D). 
A

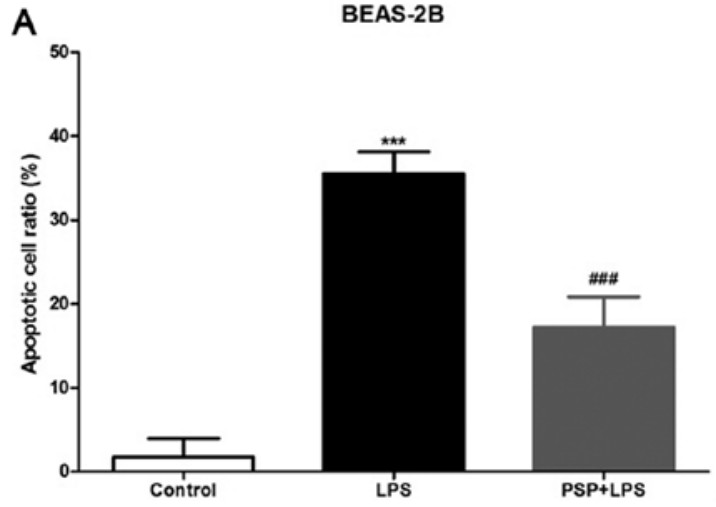

C

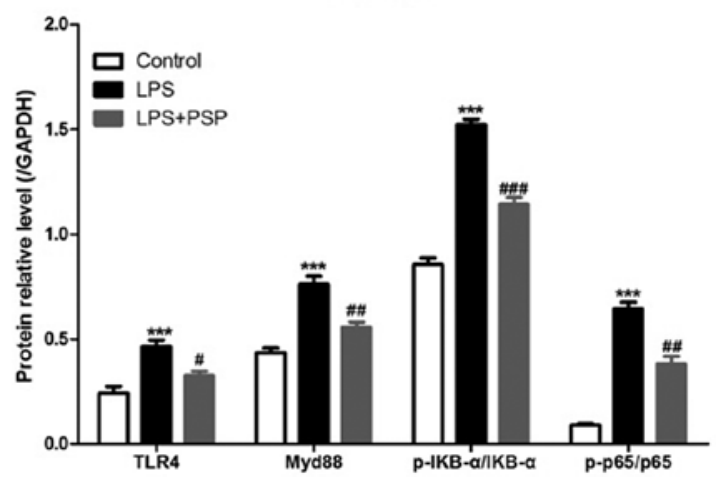

B

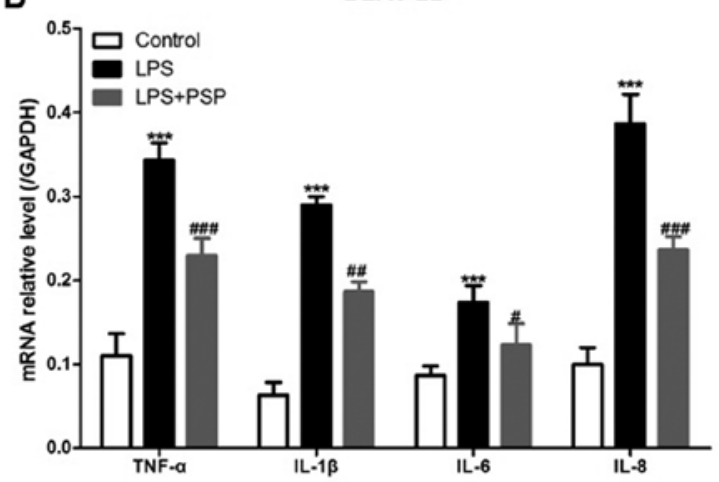

D

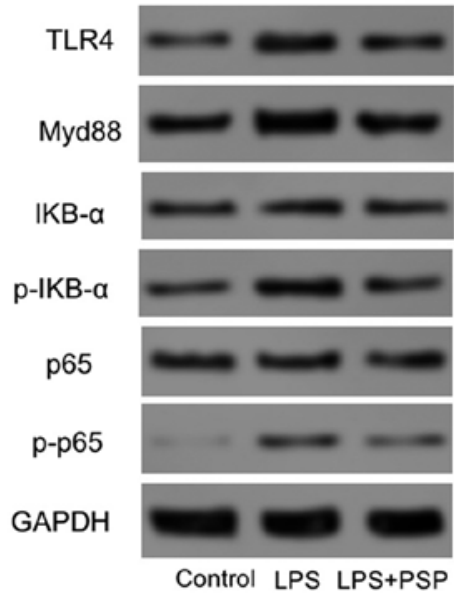

Figure 4. Effect of PSPs on LPS-induced apoptosis of BEAS-2B cells in vitro. (A) Data of BEAS-2B cell apoptosis following the different treatments. (B) Effects of the different treatments on the mRNA expression of inflammatory factors in BEAS-2B cells. (C and D) Effects of the different treatments (control group, LPS group and LPS+PSP group) on the expression of proteins in the BEAS-2B cells; At least 3 independent repetitions per experiment were conducted; ${ }^{* * *} \mathrm{P}<0.001$ vs. the control group; ${ }^{\#} \mathrm{P}<0.05,{ }^{\# \#} \mathrm{P}<0.01$ and ${ }^{\# \#} \mathrm{P}<0.001$ vs. the LPS group. PSPs, Polygonatum sibiricum polysaccharides; LPS, lipopolysaccharide.

\section{Discussion}

The main causes of acute lung injury (ALI) include infection, trauma, shock, and inhalation of toxic gases among others, and approximately 30 to $50 \%$ of cases are caused by serious infections. Endotoxin is the main pathogenic substance of bacteria, and the active ingredient of endotoxin is lipopolysaccharide (LPS), which is an important pathogenic factor of ALI $(24,25)$. Therefore, in the present study, we sought to establish a rat model for assessment of factors related to ALI by using intraperitoneal injections of LPS in combination with tracheal instillation of LPS, and by treatments with pre-intraperitoneal injection of PSPs. The results indicated that PSPs attenuated LPS-induced lung pathological changes in ALI rats, and decreased LPS-induced increases in MOP activity as well as the elevated levels of MDA in lung tissue. The occurrence of endotoxin-induced ALI involves a variety of pathophysiological mechanisms, which can be divided into classifications of direct and indirect LPS injury (such as inflammatory theory, oxidative stress and apoptosis), among which inflammatory reactions have been the most extensively studied (26). Previous research has indicated that excessive, uncontrolled, inflammatory responses are an underlying cause of ALI, and has shown that most are characterized by neutrophil dependence. Accordingly, aggregation and activa- tion of neutrophils are one of the main causes of capillary and alveolar damage in lungs $(27,28)$.

In the present study, we found that PSPs significantly decreased the LPS-induced increase in neutrophil ratio and reduced levels of inflammatory factors in the bronchoalveolar lavage fluid (BALF) of ALI rats. The activation and accumulation of neutrophils in lung tissues were found to have increased levels of pro-inflammatory cytokines, and to have caused pulmonary vascular endothelium and parenchymal cell damage by releasing toxic substances such as oxygen-free radicals, proteolytic enzymes, and arachidonic acid metabolites. Generally, ALI caused by neutrophils can be divided into several stages, including activation, accumulation, adhesion, migration, final release of inflammatory mediators, and oxygen-free radicals, and lung tissue can be protected if the drug can block any of these stages $(27,28)$. Previous research has confirmed that PSPs have antibacterial and anti-inflammatory effects $(8,9)$. An experiment using the filter paper and cup method indicated that PSPs displayed antibacterial effects against Micrococcus luteus (29), Saccharomyces cerevisiae (30) and other similar bacterial strains. Previous research also indicated that PSPs were able to control inflammation caused by bacterial infections, and that the mechanism of these effects may involve reducing the level of inflammatory mediators in the serum; however, the details of any such mechanisms remain undescribed $(8,9)$. 
In theory, regarding the underlying dynamics of inflammatory effects upon ALI, LPS enters the body and is recognized by lipopolysaccharide-binding protein (LBP), and then binds to cluster of differentiation 14 (CD14) to form the LPS/LBP/CD14 complex, which can induce inflammation through multiple signal transduction pathways to ultimately release a large amount of inflammation factors. This release causes lung damage, of which TLR4/Myd88/NF- $\kappa \mathrm{B}$ is one of the most critical pathways $(31,32)$. Our results also indicated that PSPs decreased LPS-induced increases in mRNA expression of inflammatory factors, and of TLR4, Myd88, $\mathrm{p}-\mathrm{IKB}-\alpha / \mathrm{IKB}-\alpha$ and $\mathrm{p}-\mathrm{p} 65 / \mathrm{p} 65$ proteins in lung tissue. In vitro, we found that PSPs could reduce apoptosis induced by LPS in BEAS-2B cells by inhibiting inflammation via limiting the TLR4/Myd88/NF-kB pathway.

Toll-like receptor 4 (TLR4) is the first TLR member to have been discovered, and it is now widely accepted that TLR4 is a transmembrane transduction receptor for LPS signaling from extracellular to intracellular space $(33,34)$. TLR4 directly binds to LPS and binds to LBP-LPS-CD14, which signals into the cell and initiates an inflammatory response that activates a series of downstream molecules whereby amplification of the inflammatory response occurs (34). The TLR4/NF- $\kappa B$ pathway is divided into a MyD88 (medullary differentiation protein 88)-independent pathway and a MyD88-dependent pathway. The MyD88-independent pathway is controlled by the MyD88 linker protein MAL (MyD88 linker protein), which interacts with MAL to induce activation of NF- $\mathrm{B}(35,36)$. The MyD88-dependent pathway involves the interaction of TLR4 with MyD88, whereby MyD88 is activated and phosphorylates TLR4 with an IL-1-related

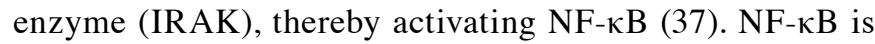
an important transcription factor that binds to the binding site of the target gene, initiates and regulates the expression of a series of inflammatory cytokines involved in the inflammatory response, participates in the inflammatory process of ALI, and mediates organ damage such as in the lungs (38). Previous research has shown that the IKK/IкB /NF- $\mathrm{KB}$ signaling pathway is an important pathway for the activation of NF- $\kappa B$ (39), in which it initiates signal transduction after LPS stimulation and activates NF- $\kappa B$. Sustained activation of NF- $\mathrm{KB}$ promotes massive cell synthesis and release of cytokines including such as TNF- $\alpha$, IL-1 $\beta$, IL-6, IL-8, IL-10, and IL-12 $(40,41)$. These cytokines directly lead to lung injury, and can also activate other signaling pathways, as well as promote expression of inflammatory factors, ultimately leading to lung injury $(42,43)$.

In conclusion, we found that PSPs were able to reduce measures of inflammation of lung tissue in ALI afflicted rats through its effects upon the TLR4/Myd88/NF- $\mathrm{B}$ pathway. Thus, PSPs are a promising potential drug that can be used for the treatment of LPS-induced ALI.

\section{Acknowledgements}

Not applicable.

\section{Funding}

No funding was recieved.

\section{Availability of data and material}

All data generated or analyzed during this study are included in this published article.

\section{Authors' contributions}

TYL and LLZ designed and performed this study, and wrote the article. TYL, SBC, BCH, JH, XH, LQ, YF and ZT carried out the data collection and analysis. All authors read and approved the manuscript and agree to be accountable for all aspects of the research in ensuring that the accuracy or integrity of any part of the work are appropriately investigated and resolved.

\section{Ethics approval and consent to participate}

The Ethics Committee of The First Affiliated Hospital of Nanchang University (Nanchang, China) has reviewed and approved this study. All the experimental procedures were carried out according to the National Institutes of Health guidelines for the use of experimental animals.

\section{Patient consent for publication}

Not applicable.

\section{Competing interests}

The authors declare that they have no competing interests.

\section{References}

1. Butt Y,Kurdowska A and Allen TC: Acute lung injury: A clinical and molecular review. Arch Pathol Lab Med 140: 345-350, 2016.

2. Rubenfeld GD and Herridge MS: Epidemiology and outcomes of acute lung injury. Chest 131: 554-562, 2007.

3. Bastarache JA, Ware LB and Bernard GR: The role of the coagulation cascade in the continuum of sepsis and acute lung injury and acute respiratory distress syndrome. Semin Respir Crit Care Med 27: 365-376, 2006.

4. Fujishima S, Morisaki H, Ishizaka A, Kotake Y, Miyaki M, Yoh K, Sekine K, Sasaki J, Tasaka S, Hasegawa N, et al: Neutrophil elastase and systemic inflammatory response syndrome in the initiation and development of acute lung injury among critically ill patients. Biomed Pharmacother 62: 333-338, 2008.

5. Nie L, Wu W, Lu Z, Zhu G and Liu J: CXCR3 may help regulate the inflammatory response in acute lung injury via a pathway modulated by IL- 10 secreted by CD $8+C D 122+$ regulatory $T$ cells. Inflammation 39: 526-533, 2016.

6. GoodmanRB,PuginJ,LeeJS and Matthay MA:Cytokine-mediated inflammation in acute lung injury. Cytokine Growth Factor Rev 14: 523-535, 2003.

7. Lin SH, Fu J, Wang CJ, Gao F, Feng XY, Liu Q, Cao J and Xu F: Inflammation elevated IL-33 originating from the lung mediates inflammation in acute lung injury. Clin Immunol 173: 32-43, 2016.

8. Cui X, Wang S, Cao H, Guo H, Li Y, Xu F, Zheng M, Xi X and Han C: A Review: The bioactivities and pharmacological applications of Polygonatum sibiricum polysaccharides. Molecules 23: $1170,2018$.

9. Li L, Thakur K, Liao BY, Zhang JG and Wei ZJ: Antioxidant and antimicrobial potential of polysaccharides sequentially extracted from Polygonatum cyrtonema Hua. Int J Biol Macromol 114: 317-323, 2018.

10. Long T, Liu Z, Shang J, Zhou X, Yu S, Tian H and Bao Y: Polygonatum sibiricum polysaccharides play anti-cancer effect through TLR4-MAPK/NF- $\mathrm{B}$ signaling pathways. Int J Biol Macromol 111: 813-821, 2018. 
11. Yang HZ, Wang JP, Mi S, Liu HZ, Cui B, Yan HM, Yan J, Li Z, Liu H, Hua F, et al: TLR4 activity is required in the resolution of pulmonary inflammation and fibrosis after acute and chronic lung injury. Am J Pathol 180: 275-292, 2012.

12. Tao H, Li N, Zhang Z, Mu H, Meng C, Xia H, Fu L, Xu Y and Zhang S: Erlotinib protects LPS-induced acute lung injury in mice by inhibiting EGFR/TLR4 signaling pathway. Shock 51: 131-138, 2019.

13. Becker CE and $\mathrm{O}^{\prime}$ Neill LAJ: Inflammasomes in inflammatory disorders: The role of TLRs and their interactions with NLRs. Semin Immunopathol 29: 239-248, 2007.

14. Lin Q, Li M, Fang D, Fang J and Su SB: The essential roles of Toll-like receptor signaling pathways in sterile inflammatory diseases. Int Immunopharmacol 11: 1422-1432, 2011.

15. Wu H, Yang Y, Guo S, Yang J, Jiang K, Zhao G, Qiu C and Deng G: Nuciferine ameliorates inflammatory responses by inhibiting the TLR4-mediated pathway in lipopolysaccharide-induced acute lung injury. Front Pharmacol 8: 939, 2017.

16. Qi M, Yin L, Xu L, Tao X, Qi Y, Han X, Wang C, Xu Y, Sun H, Liu K, et al: Dioscin alleviates lipopolysaccharide-induced inflammatory kidney injury via the microRNA let-7i/TLR4/MyD88 signaling pathway. Pharmacol Res 111: 509-522, 2016.

17. Verstak B, Nagpal K, Bottomley SP, Golenbock DT, Hertzog PJ and Mansell A: MyD88 adapter-like (Mal)/TIRAP interaction with TRAF6 is critical for TLR2- and TLR4-mediated NF-kappaB proinflammatory responses. J Biol Chem 284 24192-24203, 2009.

18. He W, Qu T, Yu Q, Wang Z, Lv H, Zhang J, Zhao X and Wang P: LPS induces IL-8 expression through TLR4, MyD88, NF-kappaB and MAPK pathways in human dental pulp stem cells. Int Endod J 46: 128-136, 2013 .

19. National Research Council (US) Committee for the Update of the Guide for the Care and Use of Laboratory Animals: Guide for the Care and Use of Laboratory Animals. Publication 327: 963-965, 2011

20. Hawkins P, Prescott MJ, Carbone L, Dennison N, Johnson C, Makowska IJ, Marquardt N, Readman G, Weary DM and Golledge HD: A good death? Report of the second Newcastle Meeting on Laboratory Animal Euthanasia. Animals (Basel) 6: $50,2016$.

21. Huang $\mathrm{CH}$, Yang ML, Tsai CH, Li YC, Lin YJ and Kuan YH: Ginkgo biloba leaves extract (EGb 761) attenuates lipopolysaccharide-induced acute lung injury via inhibition of oxidative stress and NF- $\kappa \mathrm{B}$-dependent matrix metalloproteinase-9 pathway. Phytomedicine 20: 303-309, 2013

22. Leung WS, Yang ML, Lee SS, Kuo CW, Ho YC, Huang-Liu R, Lin HW and Kuan YH: Protective effect of zerumbone reduces lipopolysaccharide-induced acute lung injury via antioxidative enzymes and $\mathrm{Nrf} 2 / \mathrm{HO}-1$ pathway. Int Immunopharmacol 46 : 194-200, 2017

23. Tao J, Zhang J, Ling Y, McCall CE and Liu TF: Mitochondrial sirtuin 4 resolves immune tolerance in monocytes by rebalancing glycolysis and glucose oxidation homeostasis. Front Immunol 9 : 419, 2018

24. Zhang B, Liu ZY, Li YY, Luo Y, Liu ML, Dong HY, Wang YX, Liu Y, Zhao PT, Jin FG, et al: Antiinflammatory effects of matrine in LPS-induced acute lung injury in mice. Eur J Pharm Sci 44: 573-579, 2011

25. Jiang W, Luo F, Lu Q, Liu J, Li P, Wang X, Fu Y, Hao K, Yan T and Ding X: The protective effect of Trillin LPS-induced acute lung injury by the regulations of inflammation and oxidative state. Chem Biol Interact 243: 127-134, 2016.

26. Wu J, Zhang YY, Guo L, Li H and Chen DF: Bupleurum polysaccharides attenuates lipopolysaccharide-induced inflammation via modulating Toll-like receptor 4 signaling. PLoS One 8: e78051, 2013

27. Caudrillier A, Kessenbrock K, Gilliss BM, Nguyen JX, Marques MB, Monestier M, Toy P, Werb Z and Looney MR: Platelets induce neutrophil extracellular traps in transfusion-related acute lung injury. J Clin Invest 122: 2661-2671, 2012.
28. Narasaraju T, Yang E, Samy RP, Ng HH, Poh WP, Liew AA, Phoon MC, van Rooijen N and Chow VT: Excessive neutrophils and neutrophil extracellular traps contribute to acute lung injury of influenza pneumonitis. Am J Pathol 179: 199-210, 2011.

29. Zhi-Tao LI, Sun JX, Zhu HX and Chu ZF: Extracting of Polygonatum polysaccharides and its antimicrobial activity. Food Res Dev 38: 36-37, 2017.

30. Zheng CY, Wang HF and Zhang TT: Studies on the anti-micromial and anti-inflammatory activities of Polygonatum cyrtonema Hua. polysaccharides. J Anhui Norm Univsity 33: 272-276, 2010 (In Chinese).

31. Jiang Q, Yi M, Guo Q, Wang C, Wang H, Meng S, Liu C, Fu Y, $\mathrm{Ji} \mathrm{H}$ and Chen T: Protective effects of polydatin on lipopolysaccharide-induced acute lung injury through TLR4-MyD88-NF- $\mathrm{B}$ pathway. Int Immunopharmacol 29: 370-376, 2015.

32. Akhter N, Hasan A, Shenouda S, Wilson A, Kochumon S, Ali S, Tuomilehto J, Sindhu S and Ahmad R: TLR4/MyD88 -mediated CCL2 production by lipopolysaccharide (endotoxin): Implications for metabolic inflammation. J Diabetes Metab Disord 17: 77-84, 2018.

33. Rocha DM, Caldas AP, Oliveira LL, Bressan $J$ and Hermsdorff HH: Saturated fatty acids trigger TLR4-mediated inflammatory response. Atherosclerosis 244: 211-215, 2016.

34. Lu YC, Yeh WC and Ohashi PS: LPS/TLR4 signal transduction pathway. Cytokine 42: 145-151, 2008.

35. Doyle SL and O'Neill LAJ: Toll-like receptors: From the discovery of NFkappaB to new insights into transcriptional regulations in innate immunity. Biochem Pharmacol 72: 1102-1113, 2006.

36. O'Neill LAJ and Bowie AG: The family of five: TIR-domain-containing adaptors in Toll-like receptor signalling. Nat Rev Immunol 7: 353-364, 2007.

37. Jiang Z, Ninomiya-Tsuji J, Qian Y, Matsumoto K and Li X: Interleukin-1 (IL-1) receptor-associated kinase-dependent IL-1-induced signaling complexes phosphorylate TAK1 and TAB2 at the plasma membrane and activate TAK1 in the cytosol. Mol Cell Biol 22: 7158-7167, 2002.

38. Monick MM and Hunninghake GW: Activation of second messenger pathways in alveolar macrophages by endotoxin. Eur Respir J 20: 210-222, 2002.

39. Perkins ND: Integrating cell-signalling pathways with NF-kappaB and IKK function. Nat Rev Mol Cell Biol 8: 49-62, 2007.

40. Dolinay T, Kim YS, Howrylak J, Hunninghake GM, An $\mathrm{CH}$, Fredenburgh L, Massaro AF, Rogers A, Gazourian L, Nakahira K, et al: Inflammasome-regulated cytokines are critical mediators of acute lung injury. Am J Respir Crit Care Med 185: 1225-1234, 2012

41. MontraversP,Chollet-MartinS,MarmuseJP,Gougerot-PocidaloMA and Desmonts JM: Lymphatic release of cytokines during acute lung injury complicating severe pancreatitis. Am J Respir Crit Care Med 152: 1527-1533, 1995.

42. Barreto TR, Costola-de-Souza C, Margatho RO, Queiroz-Hazarbassanov N, Rodrigues SC, Felício LF, Palermo-Neto J and Zager A: Repeated Domperidone treatment modulates pulmonary cytokines in LPS-induced acute lung injury in mice. Int Immunopharmacol 56: 43-50, 2018.

43. Lin S, Wu H, Wang C, Xiao Z and Xu F: Regulatory T cells and acute lung injury: Cytokines, uncontrolled inflammation, and therapeutic implications. Front Immunol 9: 1545, 2018.

(i) (2) This work is licensed under a Creative Commons Attribution-NonCommercial-NoDerivatives 4.0 International (CC BY-NC-ND 4.0) License. 